\title{
NUMERICAL SOLUTIONS FOR SPACE FRACTIONAL DISPERSION EQUATIONS WITH NONLINEAR SOURCE TERMS
}

\author{
Hong Won Choi, Sang Kwon Chung, and Yoon Ju Lee
}

\begin{abstract}
Numerical solutions for the fractional differential dispersion equations with nonlinear forcing terms are considered. The backward Euler finite difference scheme is applied in order to obtain numerical solutions for the equation. Existence and stability of the approximate solutions are carried out by using the right shifted Grünwald formula for the fractional derivative term in the spatial direction. Error estimate of order $O(\Delta x+\Delta t)$ is obtained in the discrete $L_{2}$ norm. The method is applied to a linear fractional dispersion equations in order to see the theoretical order of convergence. Numerical results for a nonlinear problem show that the numerical solution approach the solution of classical diffusion equation as fractional order approaches 2 .
\end{abstract}

\section{Introduction}

The fractional order diffusion equations have been discussed by many authors as generalizations of classical diffusion equation in order to treat suband super-diffusive processes. The equation having these anomalous diffusion models in applications fluid flow, finance, biological sciences and others $[1,6]$.

In this paper we will discuss numerical approximate solutions for the fractional differential dispersion equation with a nonlinear forcing term. The equation is described as

$$
\frac{\partial u(x, t)}{\partial t}=\frac{\partial^{\alpha} u(x, t)}{\partial x^{\alpha}}+f(u, x, t), \quad(x, t) \in \Omega \times(0, T] .
$$

Here $f(u, x, t)$ is globally Lipschitz continuous with the maximum sustainable density $K$ of $u$ which is a natural assumption in biology. And $T$ is a positive

Received April 14, 2009.

2000 Mathematics Subject Classification. 65N06, 65N12, 65N15, 65R20.

Key words and phrases. fractional differential equation, Riemann-Liouville fractional derivative, Caputo fractional derivative, finite difference scheme, stability, convergence, error estimate.

The second author is supported by Education and Research Foundation of College of Education, Seoul National University. 
real number as a terminal time. For additional restrictions we need Dirichlet boundary conditions

$$
u(0, t)=0, \quad u(1, t)=0, \quad t \in(0, T] .
$$

The boundary condition indicates that the left boundary and the right boundary are far away enough from an evolving particle plume, so that no significant concentrations reach the boundary. Furthermore, the initial condition is given as

$$
u(x, 0)=u_{0}(x), \quad x \in \bar{\Omega} .
$$

The values $\alpha, 1<\alpha<2$, model a super diffusive process in the spatial direction and the model with $\alpha=2$ describes the classical dispersion. In (1.1) the Riemann-Liouville fractional derivative of order $\alpha$ is defined as

$$
\frac{\partial^{\alpha} \phi(x, t)}{\partial x^{\alpha}}=\frac{1}{\Gamma(n-\alpha)} \frac{d^{n}}{d x^{n}} \int_{0}^{x} \frac{\phi(\xi)}{(x-\xi)^{\alpha+1-n}} d \xi,
$$

where $n$ is an integer such that $n-1<\alpha<n$ and $\Gamma(\cdot)$ is the gamma function:

$$
\Gamma(\alpha)=\int_{0}^{\infty} e^{-x} x^{\alpha-1} d x .
$$

The theoretical results on unique existence of the solution for (1.1) have been studied by Baeumer, Kovács and Meerschaert [2] using the semigroup theory when $f$ is globally Lipschitz continuous. Numerical solutions are obtained in [2] by applying the operator splitting methods based on the theory of operator semigroups. It needs to find the solution operators via convolution formula and then numerical computations.

The finite difference numerical approximation to the problem (1.1)-(1.3) with Riemann-Liouville fractional derivative in one space dimension has been discussed by Meerschaert and Tadjeran [6] when $f(u)=f(x, t)$. They used the Euler scheme with the shifted Grünwald estimate to the fractional derivative and showed that the explicit scheme is conditionally stable but the implicit scheme is unconditionally stable using Gerschigorin's circle theorem. Tadjeran, Meerschaert and Scheffler [11] have obtained a second order accuracy $O\left(\Delta x+(\Delta t)^{2}\right)$ by using the Crank-Nicolson difference scheme for $f(u)=$ $f(x, t)$. Numerical computations without any theoretical discussions are carried out by Lynch, Carreras, del-Castillo-Negrete, Ferreira-Mejias, and Hicks [4] by using semi-implicit difference methods and compared numerical results.

For a two-dimensional problem with fractional Riemann-Liouville derivative when $f(u)=f(x, y, t)$, Meerschaert, Scheffler and Tadjeran [7] used an implicit Euler difference scheme. They obtained consistency and convergence of order $O(\Delta x+\Delta t)$ and applied alternating-direction implicit (ADI) method for numerical computation. Finite element methods have been also applied to the fractional advection diffusion equations by Roop [10] and Ervin, Heuer and Roop [3] when $f(u)=f(x, y, t)$. 
The above finite difference methods have been applied only to the linear problems. The layout of this paper is as follows. Basic notations and some preliminary results are introduced and a finite difference scheme with Grünwald estimate for the Volterra integral for the problem is given in Section 2. In Section 3, existence of approximate solutions will be given using Leray-Schauder fixed point theorem. Consistency, stability and error estimates of the scheme are also considered. In Section 4, numerical experiments are carried out to see convergence of the scheme.

\section{Finite difference approximations}

Since it is known in [5] that the unshifted Grünwald formula for the fractional derivative of Riemann-Liouville type is unstable regardless a finite difference scheme is either explicit or implicit, we adopt the right-shifted Grünwald formula to the equation (1.1). The the right-shifted Grünwald formula for $1<\alpha<2$ is given by

$$
\frac{\partial^{\alpha} u(x, t)}{\partial x^{\alpha}}=\frac{1}{\Gamma(-\alpha)} \lim _{N \rightarrow \infty} \frac{1}{h^{\alpha}} \sum_{k=0}^{N} \frac{\Gamma(k-\alpha)}{\Gamma(k+1)} u(x-(k-1) h, t)+O(h),
$$

where $N$ is a positive integer. For simple notations we define the normal like Grünwald weights by

$$
g_{\alpha, k}=\frac{\Gamma(k-\alpha)}{\Gamma(-\alpha) \Gamma(k+1)}=(-1)^{k}\left(\begin{array}{l}
\alpha \\
k
\end{array}\right)=(-1)^{k} \frac{\alpha(\alpha-1) \cdots(\alpha-k+1)}{k !}
$$

For example $g_{\alpha, 0}=1, g_{\alpha, 1}=-\alpha$. It is well known that

$$
\sum_{k=0}^{M-1} \frac{\Gamma(k-\alpha)}{\Gamma(-\alpha) \Gamma(M)}=\frac{\Gamma(M-\alpha)}{\Gamma(-\alpha) \Gamma(M)}
$$

for any positive integer $M[8]$.

For a finite difference numerical approximation scheme, define $t_{n}=n \Delta t$ with $0 \leq t_{n} \leq T$. Let $h=\Delta x$ be the grid size in the spatial direction with $\Delta x=1 / N$ and $x_{i}=i \Delta x$ for $i=0,1,2, \ldots, N$. Define $u_{i}^{n}$ as the numerical approximation to $u\left(x_{i}, t_{n}\right)$ and similarly $f_{i}^{n}$ denotes the approximation to $f\left(u\left(x_{i}, t_{n}\right)\right)$. For a discretization in the temporal direction, $\frac{\partial u}{\partial t}$ is approximated by

$$
\partial_{t} u^{n}=\frac{u^{n}-u^{n-1}}{\Delta t} .
$$

Then the implicit Euler approximation for (1.1) with the right shifted Grünwald estimates becomes

$$
\partial_{t} u^{n}=\frac{1}{h^{\alpha}} \sum_{k=0}^{i+1} g_{\alpha, k} u_{i-k+1}^{n+1}+f_{i}^{n+1}
$$


Hence we have to find $u_{i}^{n}, i=0,1,2, \ldots, N, n=0,1, \ldots$, satisfying

$$
u_{i}^{n+1}=u_{i}^{n}+\frac{\Delta t}{h^{\alpha}} \sum_{k=0}^{i+1} g_{\alpha, k} u_{i-k+1}^{n+1}+\Delta t f_{i}^{n+1} .
$$

We introduce a discrete inner product and the induced discrete norm, respectively, as

$$
(u, v)=h \sum_{k=0}^{N} u_{i} v_{i}, \quad\|u\|=(u, u)^{1 / 2}
$$

for $u=\left(u_{0}, u_{1}, u_{2}, \ldots, u_{N}\right)$ and $v=\left(v_{0}, v_{1}, v_{2}, \ldots, v_{N}\right)$.

\section{Stability and convergence}

In order to show existence of solutions for (2.4), we recall Leray-Schauder fixed point theorem [9].

Lemma 3.1. For a given open and bounded domain $D \subset \mathbb{R}^{n}$ containing the origin $0 \in D$, let $F: D \rightarrow \mathbb{R}^{n}$ be a continuous function. If $F(x) \neq r x$ for all $r>1$ and $x \in D$, then $F$ has a fixed point in $\bar{D}$ which is the closure of $D$.

We may show that the finite difference scheme for (2.1) has a solution.

Theorem 3.1. The equation (2.4) has a solution.

Proof. Consider a ball $D=B(0, a) \subset \mathbb{R}^{n}$ centered at the origin for a positive constant $a$ and let $\partial D$ be the boundary of $D$. Suppose there exists a vector $u=\left(u_{0}, u_{1}, u_{2}, \ldots, u_{N}\right) \in \partial D$ such that for some $\gamma>1$

$$
\gamma u=F(u)+u^{n},
$$

where

$$
F_{i}(u)=\frac{\Delta t}{h^{\alpha}} \sum_{k=0}^{i+1} g_{\alpha, k} u_{i-k+1}+\Delta t f\left(u_{i}\right) .
$$

Taking a discrete inner product of (3.1) with $u$, we obtain

$$
\begin{aligned}
\gamma\|u\|^{2} & =(F(u), u)+\left(u^{n}, u\right) \\
& =\frac{\Delta t}{h^{\alpha}}(A u, u)+\Delta t(f(u), u)+\left(u^{n}, u\right),
\end{aligned}
$$

where an $(N+1) \times(N+1)$ matrix $A=\left(a_{i j}\right)$ is given by

$$
a_{i j}=\left\{\begin{aligned}
a_{i i} & =g_{\alpha, 1}, \quad i=1, \ldots, N+1 \\
a_{i, i+1} & =g_{\alpha, 0}, \quad i=1, \ldots, N \\
a_{i, j} & =0, \quad i=1, \ldots, N+1, j=i+2, i+3, \ldots, N+1 \\
a_{i, j} & =g_{\alpha, i+1-j}, \quad j=0,1, \ldots, i-1
\end{aligned}\right.
$$


Since $f(u, x, t)$ is continuous on $[-K, K] \times[0,1] \times[0, T]$, we may assume that $\|f\|_{\infty} \leq B$ for some positive constant. It follows from applying Schwarz inequality that

$$
\gamma\|u\|^{2} \leq \frac{\Delta t}{h^{\alpha}}\|A\|\|u\|^{2}+B \Delta t\|u\|^{2}+\left\|u^{n}\right\|\|u\|,
$$

where the matrix norm $\|A\|=\sup _{x \neq 0} \frac{\|A\|}{\|x\|}$ for $x \in \mathbb{R}^{N+1}$. Since $1<\alpha<2$ and $\Gamma(-\alpha)=\frac{-\pi \csc (\pi x)}{\Gamma(\alpha+1)}$, it follows from $(2.3)$ that $\|A\|$ is bounded by a positive number. If we divide both sides by $\|u\|^{2}$, then

$$
\gamma \leq\left(\frac{\|A\|}{h^{\alpha}}+B\right) \Delta t+\frac{\left\|u^{n}\right\|}{\|u\|} .
$$

If we take $\|u\|$ sufficiently large and $\Delta t$ small enough, then $\gamma \leq 1$. This leads to a contradiction. Hence

$$
\gamma u \neq F(u)+u^{n}, \quad \forall \gamma>1, \quad u \in \partial D .
$$

Thus there is a solution $u \in \bar{D}$ such that

$$
u=F(u)+u^{n} .
$$

This completes the proof.

Theorem 3.2. The approximate scheme (2.4) is stable.

Proof. It follows from the equation (2.4) that, by taking an inner product,

$$
\partial_{t}\left\|u^{n}\right\|^{2} \leq \frac{\|A\|}{h^{\alpha}}\left\|u^{n}\right\|^{2}+B\left\|u^{n}\right\|^{2} .
$$

Hence

$$
\left\|u^{n}\right\|^{2}-\left\|u^{n-1}\right\|^{2} \leq \frac{\Delta t}{h^{\alpha}}\|A\|\left\|u^{n}\right\|^{2}+B \Delta t\left\|u^{n}\right\|^{2} .
$$

Summing from $n=1$ to $n$, we obtain

$$
\left\|u^{n}\right\|^{2} \leq\left\|u^{0}\right\|^{2}+\Delta t\left(\frac{\|A\|}{h^{\alpha}}+B\right) \sum_{k=1}^{n}\left\|u^{k}\right\|^{2} .
$$

By applying Gronwall's inequality, we obtain

$$
\left\|u^{n}\right\|^{2} \leq C\left\|u^{0}\right\|^{2},
$$

where the generic constant $C$ is independent of $\Delta t$. This completes the proof.

Remark 3.1. Uniqueness of the solution for (2.4) can be shown easily from the above theorem.

We now consider the error estimates of the approximation scheme (2.4).

Theorem 3.3. The approximation scheme (2.4) for (1.1) is convergent of order $O(h+\Delta t)$. 
Proof. Let $U^{n}$ denote the exact solution $u\left(\cdot, t_{n}\right)$ of the approximation scheme (2.4). And let $e^{n}=U^{n}-u^{n}$ be the error. Then

$$
\partial_{t} e_{i}^{n}=\frac{1}{h^{\alpha}} \sum_{k=1}^{i+1} g_{\alpha, k} e_{i-k+1}^{n}+f_{i}\left(U^{n}\right)-f_{i}\left(u^{n}\right)+R_{i}\left(U^{n}\right),
$$

where $R_{i}(u)=O(h+\Delta t)$ which is obtained by Taylor's expansion. We now assume that $f$ has a Lipschitz constant $L$ so that

$$
|f(U, x, t)-f(u, x, t)| \leq L\|U-u\| .
$$

Taking an inner product with $e^{n}$ and summing from $n=1$ to $n$, then

$$
\left\|e^{n}\right\|^{2} \leq\left\|e^{0}\right\|^{2}+\left(\frac{\|A\|}{h^{\alpha}}+L\right) \Delta t \sum_{j=1}^{n}\left\|e^{j}\right\|^{2}+\Delta t \sum_{j=1}^{n}\left(R\left(U^{j}\right), e^{j}\right) .
$$

Since $\left\|e^{0}\right\|=0$, it follows from the Schwarz inequality and Young's inequality that

$$
\begin{aligned}
\left\|e^{n}\right\|^{2} & \leq\left(\frac{\|A\|}{h^{\alpha}}+L\right) \Delta t \sum_{j=1}^{n}\left\|e^{j}\right\|^{2}+\Delta t \sum_{j=1}^{n} C(h+\Delta t)\left\|e^{j}\right\| \\
& \leq C(h+\Delta t)^{2}+\left(1+L+\frac{\|A\|}{h^{\alpha}}\right) \Delta t \sum_{j=1}^{n}\left\|e^{j}\right\|^{2} .
\end{aligned}
$$

Now it follows from Grownwall's inequality [12] that

$$
\left\|e^{n}\right\|^{2} \leq C(h+\Delta t)^{2} .
$$

This completes the proof.

Remark 3.2. A second-order accuracy in the temporal direction has been obtained by Tadjeran, Meerschaert, and Scheffler [11] for the linear problem when $f(u, x, t)=f(x, t)$. We may obtain the order of convergence $O\left(h+(\Delta t)^{2}\right)$ for the Crank-Nicolson method following the ideas of the backward Euler method.

\section{Numerical experiments}

In order to see the implementation of the previous theoretical results, we consider two examples. Since, as in [5],

$$
-a_{i i}=-g_{\alpha, 1}=\alpha \geq \sum_{k=0, k \neq 1}^{i+1} g_{\alpha, k}=\sum_{j=0, j \neq i}^{N+1}\left|a_{i j}\right|, \quad i=0,1, \ldots, N+1,
$$

the matrix $I-\frac{\Delta t}{h^{\alpha}} A$ is irreducible and strictly diagonally dominant. Hence we may solve the system numerically by using the successive over-relaxation method [13]. Furthermore, since the spectral radius $\rho\left(\left(I-\frac{\Delta t}{h^{\alpha}} A\right)^{-1}\right)<1$, the method is unconditionally stable. 
Example 4.1. We first consider a linear fractional diffusion equation as in [11]

$$
\frac{\partial u}{\partial t}=d(x) \frac{\partial^{\alpha} u}{\partial x^{\alpha}}+q(x, t), \quad 0<x<1
$$

with the diffusion coefficient $\alpha=1.8$ and

$$
d(x)=\Gamma(2.2) x^{2.8} / 6=0.183634 x^{2.8},
$$

the source term

the initial condition

$$
q(x, t)=-(1+x) e^{-t} x^{3}
$$

and the boundary conditions

$$
u(x, 0)=x^{3}, \quad 0<x<1,
$$

$$
u(0, t)=0, \quad u(1, t)=e^{-t} .
$$

In this case the exact solution for the equation (4.2) is

$$
u(x, t)=e^{-t} x^{3} .
$$

If we discretize the equation (4.2), we obtain a system of linear equations with a strictly diagonally dominant matrix. We may solve the system by using the successive over-relaxation method. Table 1 shows that the numerical convergence is of order $O(\Delta x+\Delta t)$. And Figure 1 shows the maximum error for the equation (4.2) when $t=1.0$.

TABle 1. Convergence for (4.2)

\begin{tabular}{|c|c|c|c|}
\hline$\Delta x$ & $\Delta h$ & $\|e\|_{\infty}$ & Convergence order \\
\hline$\frac{1}{10}$ & $\frac{1}{10}$ & 0.008055657 & - \\
\hline$\frac{1}{15}$ & $\frac{1}{15}$ & 0.005485266 & 1.47 \\
\hline$\frac{1}{20}$ & $\frac{1}{20}$ & 0.004241183 & 1.29 \\
\hline$\frac{1}{40}$ & $\frac{1}{40}$ & 0.002380952 & 1.78 \\
\hline
\end{tabular}

Example 4.2. We now consider a Fisher-Kolmogorov equation with fractional diffusion term of order $1<\alpha<2$ as

$$
\frac{\partial u}{\partial t}=d \frac{\partial^{\alpha} u}{\partial x^{\alpha}}+r u\left(1-\frac{u}{K}\right)
$$

with an initial condition

$$
u(x, 0)=u_{0}(x)
$$

and boundary conditions

$$
u(-100, t)=0, \quad u(100, t)=0 .
$$

If we discretize the equation (4.3), we obtain a system of nonlinear equations with an irreducibly diagonally dominant matrix. We may solve the system by using the generalized Newton's method. Numerical computation is performed with $K=1, d=0.1, r=0.25$ and $u_{0}(x)=1.0$ around the origin and rapidly 


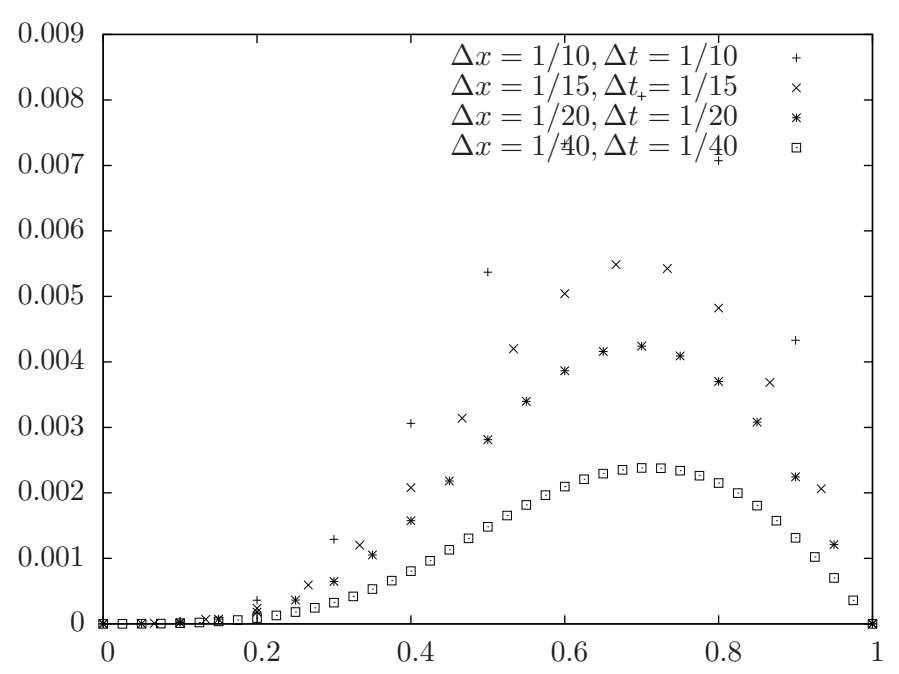

Figure 1. Errors for (4.2)

decays to 0 away from the origin as in [1]. Figure 2 shows the numerical solutions at $t=30.0$, which converge to the solution of classical dispersion equation as $\alpha \rightarrow 2$.

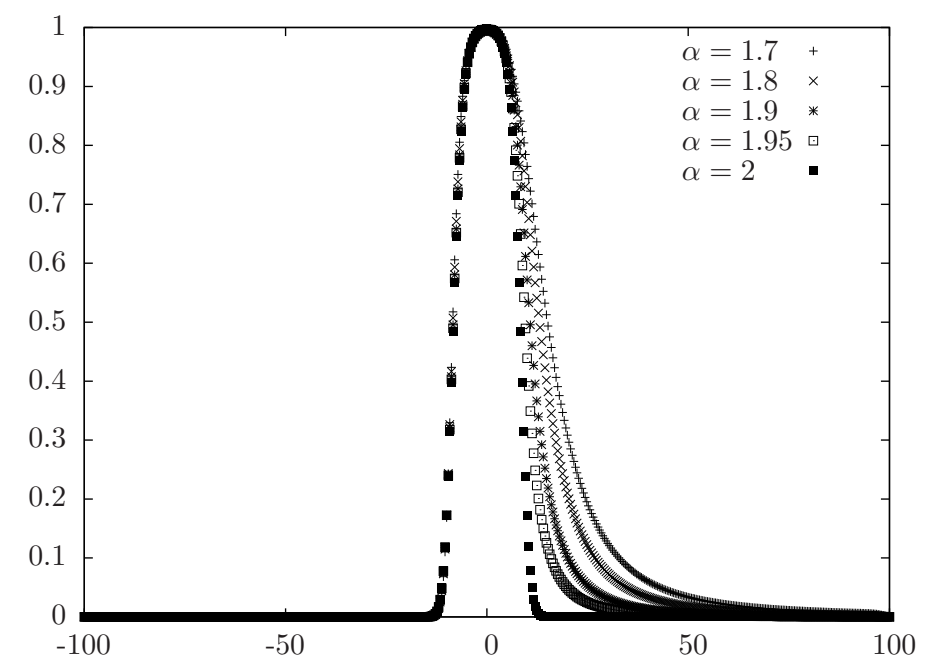

FiguRE 2. Numerical solutions for (4.3) 


\section{Concluding remarks}

In this paper we have discussed numerical solutions for space fractional dispersion equations with a source term. We have applied the backward Euler finite difference method with the right-shifted Grüwald formula for the RiemannLiouville space fractional derivative term. Leray-Schauder fixed point theorem has been used for existence of solution for the method and the stability and error estimates of order $O(\Delta x+\Delta t)$ are considered. Numerical computations are performed using the generalized Newton's method because the coefficient matrix is strictly diagonally dominant. Numerical results show that the solution of dispersion equation with a fractional derivative converges to that of the classical dispersion equation as the fractional order approaches 2.

It is necessary to develop an explicit finite difference method which is of higher order convergence since an implicit method needs large memory due to the fractional derivative. This will be done elsewhere.

\section{References}

[1] B. Baeumer, M. Kovács, and M. M. Meerschaert, Fractional reproduction-dispersal equations and heavy tail dispersal kernels, Bull. Math. Biol. 69 (2007), no. 7, 2281-2297.

[2] , Numerical solutions for fractional reaction-diffusion equations, Comput. Math. Appl. 55 (2008), no. 10, 2212-2226.

[3] V. J. Ervin, N. Heuer, and J. P. Roop, Numerical approximation of a time dependent, nonlinear, space-fractional diffusion equation, SIAM J. Numer. Anal. 45 (2007), no. 2, $572-591$.

[4] V. E. Lynch, B. A. Carreras, D. del-Castillo-Negrete, K. M. Ferreira-Mejias, and H. R. Hicks, Numerical methods for the solution of partial differential equations of fractional order, J. Comput. Phys. 192 (2003), no. 2, 406-421.

[5] M. M. Meerschaert and C. Tadjeran, Finite difference approximations for fractional advection-dispersion flow equations, J. Comput. Appl. Math. 172 (2004), no. 1, 65-77.

[6] __ Finite difference approximations for two-sided space-fractional partial differential equations, Appl. Numer. Math. 56 (2006), no. 1, 80-90.

[7] M. M. Meerschaert, H.-P. Scheffler, and C. Tadjeran, Finite difference methods for two-dimensional fractional dispersion equation, J. Comput. Phys. 211 (2006), no. 1, 249-261.

[8] K. B. Oldham and J. Spanier, The Fractional Calculus, Dover Publications, New York, 2002.

[9] J. M. Ortega and W. C. Rheinboldt, Iterative Solution of Nonlinear Equations in Several Variables, Academic Press, New York-London, 1970.

[10] J. P. Roop, Computational aspects of FEM approximation of fractional advection dispersion equations on bounded domains in $\mathbb{R}^{2}$, J. Comput. Appl. Math. 193 (2006), no. $1,243-268$.

[11] C. Tadjeran, M. M. Meerschaert, and H.-P. Scheffler, A second-order accurate numerical approximation for the fractional diffusion equation, J. Comput. Phys. 213 (2006), no. 1, 205-213.

[12] H. Ye, J. Gao, and Y. Ding, A generalized Gronwall inequality and its application to a fractional differential equation, J. Math. Anal. Appl. 328 (2007), no. 2, 1075-1081.

[13] R. S. Varga, Matrix Iterative Analysis, Prentice-Hall, Inc., Englewood Cliffs, N.J., 1962. 
Hong Won ChOI

Department of Mathematics

Seoul Science High School

SEOUl 110-530, KoreA

E-mail address: gaul@dreamwiz.com

SAng Kwon Chung

Department of Mathematics Education

Seoul National University

SEOUl 151-748, KoreA

E-mail address: chung@snu.ac.kr

YOON JU LEE

Department of Mathematics

Seoul Science High School

Seoul 110-530, Korea

E-mail address: leeyoonju@empal.com 